\title{
High Volume Evacuator (HVE) in reducing aerosol- an exploration worth by clinicians
}

\begin{abstract}
Dentists and dental hygienists deploying air-turbine hand pieces during cavity cutting, ultrasonic scalers and high-speed hand pieces get themselves exposed to air-borne contaminants like aerosols and splatter. Mucosal and nasal inhalation makes them susceptible to tuberculosis, hepatitis B \& HIV because of established contact with respiratory secretions, saliva and blood splatter. Infection control practices have been advocated to prevent and minimize the exposure to aerosols through use of rubber dams, saliva ejectors, and pre-procedural rinse with $0.2 \%$ chlorhexidine before ultrasonic scaling. Universal precautions already in practice in dental establishments using gloves, protective eyewear with solid-side shields, face masks/chin-length plastic face shields, protective clothing (Aprons, Gowns, Clinic Jackets, Lab coats and Uniforms are helpful in containment of aerosols. High Volume Evacuator devices have been found to be efficient in substantive reduction of aerosols and should be exercised in routine dental procedures
\end{abstract}

Keywords: dentists, aerosols, infection control
Volume 9 Issue 3 - 2018

\begin{abstract}
Avijit Avasth
Department of Public Health Dentistry, Maharishi

Markandeshwar College of Dental Sciences and Research, India

Correspondence: Avijit Avasthi, Lecturer, Department of Public Health Dentistry, Maharishi Markandeshwar College of Dental Sciences and Research, Ambala, Haryana, India, Tel +917837660552, Email avijit|23avasthi@gmail.com
\end{abstract}

Received: April 06, 2018 | Published: May 16, 2018

\section{Introduction}

Dentists working in routine dental procedures involving ultrasonic scalers, high-speed hand pieces in endodontic procedures and prophylaxis cup-polishing, get exposed to hazardous airborne particles like aerosols, splatter, droplet nuclei and particulate matter. ${ }^{1}$ Aersols are generated when air, water spray and air turbine hand pieces are used; they may contain up to 100,000 bacteria per cubic foot of air and remain airborne for long time. ${ }^{2}$ Aerosol and splatter composed of blood, bacteria, saliva and tissue fluid cause exposure to blood-borne pathogens. Accidental inhalation is responsible for dissemination of pathogenic microorganisms which eventually have caused diseases tuberculosis, hepatitis-B, HIV and Severe Acute Respiratory syndrome (SARS) among dentists and dental hygienists. ${ }^{3,4}$

\section{Precautions \& interventions in controlling aerosols}

Centre for Disease Control \& American Dental Association Infection Control Guidelines have been mandating universal precautions such as gloves, protective eyewear with solid-side shields, face masks/chin-length plastic face shields, protective clothing (Aprons, Gowns, Clinic Jackets, Lab coats and Uniforms to minimize the contact with aerosols in dentists. ${ }^{4}$ Pre-procedural rinse with $0.2 \%$ chlorhexidine before ultrasonic scaling, ${ }^{1,2}$ using isolation devices (rubber dams), saliva ejectors \& HVEs have been tested in controlling aerosol production in dental settings and studies have shown promising results with HVEs causing $90 \%$ to $98 \%$ reduction of aerosols irrespective of source. ${ }^{5}$

\section{Role of high- volume evacuator}

A high-volume evacuator is a suction device that draws a large volume of air over a period of time and is fitted on to an evacuation system that is said to remove a volume of air up to 100 cubic feet per minute. HVE may address aerosol reduction but certain technical specifications are to be considered by clinicians in using HVE. a. Clinicians need to check the power and airflow volume of the HVE periodically. There are systems which have clean lines and show sufficient airflow but may have an extremely low static measurement of vacuum pressure $(\mathrm{mmHg})$. This results in backflow. There might be, an evacuation system showing high static reading but have clogged lines, resulting in low volume. In multichair dental clinics where large number of dental operators are working on a suction system loop, there is reduction in volume and pressure. Predominantly suction systems are able to remove pooling water, but sometimes waterlines may get clogged, and reduce suction volume performance.

b. Proper distance should be maintained by clinicians while holding HVE devices. The device should be held approximately $6-15 \mathrm{~mm}$ away from the active ultrasonic tip or air polisher. ${ }^{6}$

c. Clinicians need to comfortably access the mouth when using the HVE. The angulation of HVE device into patient's mouth should be done to avoid contact with cheek/ tongue of the patient.

d. There are ergonomic limitations for clinicians since they might face difficulty in holding the HVE which are heavy to handle; inability to view mouth in direct vision.

e. There is answer in resolving the difficulties faced by the dentists by using High volume Evacuation Mirror system designed by $\mathrm{Nu}$ Bird (Figure 1).

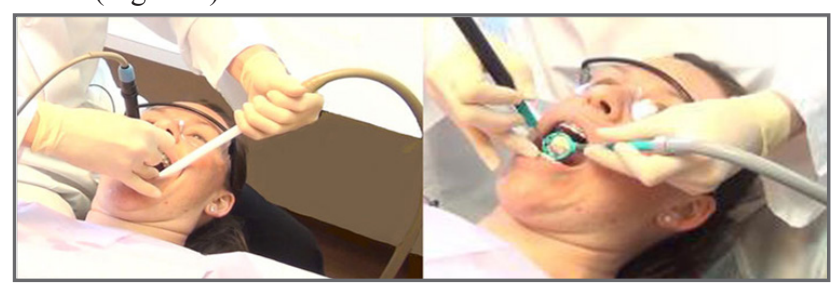

Figure I Aerosol and water management using $\mathrm{HV}$ evacuation mirror technology 
The High Volume Evacuation System designed by $\mathrm{Nu}$ bird offers mobility, easy adaptability in all areas of mouth. The Broadsweep suction system reduces device from sucking up tissue; easy light weight handling and multifunctional capability allows smooth completion of dental procedures. It comes at a price of US 219 dollars. However it is important for clinicians to conduct tests, monitor and maintain the vacuum systems used in dental offices by following manufacturer's instructions. Hence in routine dental practice the health and safety of dentists, dental hygienists and other team staff should be of paramount importance.

\section{Acknowledgements}

None.

\section{Conflict of interest}

The author declares that there is no conflict of interest

\section{References}

1. sHarrel SK, Barnes JSB, Hidalgo FR. Aerosol and Splatter Contamination from Operative Site During Ultrasonic Scaling. J Am Dent Assoc. 1998;129(9):1241-9.
2. Logotothetis DD, Welles-Martinez JM. Reducing Bacterial Aerosol Contamination with a Chlorhexidine Gluconate Pre-Rinse. J Am Dent Assoc. 1995;126(12):1634-9.

3. Bentley CD, Burkhart NW, Crawford JJ. Evaluating Splatter and Aerosol Contamination During Dental Procedures. J Am Dent Assoc. 1994;125(5):579-84.

4. LI RWK, Leung KWC, Sun FCS, et al. Severe Acute Respiratory Syndrome (SARS) and the GDP. Part II: Implications for GDPs. British Dental Journal. 2004;197(3):130-134.

5. Infection control recommendations for dental office and dental laboratory ADA Council on Scientific Affairs and ADA Council on Dental Practice. $J$ Am Dent Assoc. 1996;127(5):672-680.

6. Laura Emmons RDH, Cheri Wu, Tia Shutter. High-volume evacuation: Aerosols - it's what you can't see that can hurt you. 2017. 